\title{
Prevalencia de Streptococcus beta hemolítico en pacientes con faringoamigdalitis aguda, en un hospital de la ciudad de Chachapoyas, Amazonas
}

\author{
Prevalence of beta hemolytic Streptococcus in patients with acute pharyngoamigdalitis in \\ a Chachapoyas, Amazonas hospital
}

\author{
José María Guevara D 1, José Aguirre ${ }^{2}$, Esther Valencia ${ }^{1}$, José María Guevara G ${ }^{1}$, \\ Fernando Williams ${ }^{2}$, Elizabeth Cuéllar ${ }^{2}$, Mirtha Barboza ${ }^{2}$, Wini Agurto ${ }^{2}$ \\ ${ }^{1}$ Instituto de Medicina Tropical Daniel A. Carrión, Facultad de Medicina, Universidad Nacional Mayor de San Marcos. Lima, Perú. \\ ${ }^{2}$ Hospital I Higos Urco, EsSalud. Chachapoyas, Perú.
}

\begin{abstract}
Resumen
Introducción: En Chachapoyas hay numerosos pacientes con faringoamigdalitis aguda y cuadros clínicos con las complicaciones no supurativas que causa el Streptococcus pyogenes. Diseño: Estudio transversal. Lugar: Hospital I Higos Urco, EsSalud, Chachapoyas, Amazonas, e Instituto de Medicina Tropical Daniel A. Carrión, Universidad Nacional Mayor de San Marcos. Participantes: Pacientes con faringoamigdalitis aguda. Intervenciones: A 148 pacientes, seleccionados aleatoriamente, que acudieron al consultorio externo de otorrinolaringología por presentar cuadros clínicos compatibles con faringoamigdalitis aguda, se les tomó muestras de secreción faringoamigdaliana con hisopos y, usando el medio de transporte Amies con carbón (Difco), fueron enviados al Instituto de Medicina Tropical Daniel A. Carrión, en donde fueron procesados. Principales medidas de resultados: Presencia de Streptococcus beta hemolítico y otras bacterias cultivables. Resultados: Las enterobacterias fueron las más aisladas $(49,1 \%)$ de los cultivos positivos. Solo 5 Streptococcus beta hemolíticos fueron aislados: un Streptococcus pyogenes, tres Streptococcus agalactiae y un Streptococcus del grupo G, los cuales fueron sensibles a los betalactámicos, macrólidos y lincosamidas. Conclusiones: Se sugiere realizar estudios complementarios con el dosaje de antiestreptolisina 0.
\end{abstract}

Palabras clave: Tonsila faríngea; Streptococcus pyogenes; antiestreptolisina.

\begin{abstract}
Introduction: There are numerous Chachapoyas patients with acute pharyngoamigdalitis and clinical non suppurative complications caused by Streptococcus pyogenes. Design: Transversal study. Setting: Hospital I Higos Urco, EsSalud, Chachapoyas, Amazonas, and Instituto de Medicina Tropical Daniel A. Carrion, Universidad Nacional Mayor de San Marcos. Participants: Patients with acute pharyngoamigdalitis. Interventions: One hundred and forty-eight randomized outpatient subjects attending an ear, nose and throat office for clinical symptoms compatible with acute pharyngoamigdalitis had a sample of pharynx and tonsils taken with a cotton swab and sent in Amies with carbon medium (Difco) to the Instituto de Medicina Tropical Daniel A. Carrion to be processed. Main outcome measures: Presence of beta hemolytic Streptococcus and other bacteriae. Results: Positive cultures revealed mainly Enterobacteriae $(49,1 \%)$. Only 5 beta hemolytic Streptococcus were isolated: one Streptococcus pyogenes, three Streptococcus agalactiae and one group G Streptococcus, all sensitive to betalactamics, macrolides and lincosamides. Conclusions: We suggest to do complementary studies with antiestreptolysin 0 determination.
\end{abstract}

Key words: Adenoids; Streptococcus pyogenes; antistreptolysin.

\section{INTRODUCCIÓN}

Las infecciones faringo-amigdalianas son procesos patológicos muy frecuentes en el hombre, presentándose al menos una vez durante toda la vida.

Se reconoce al Streptococcus beta hemolítico grupo A (Streptococcus pyogenes) como el principal agente etiológico bacteriano de la faringoamigdalitis aguda, pudiendo ocasionar en la población infantil una infección aguda sin complicación, una epidemia o desencadenar una complicación tardía.

La faringoamigdalitis es una enfermedad frecuente en la infancia; su prevalencia es mayor entre los 5 y los 15 años de edad, alcanzando su pico más elevado durante los primeros 5 años de escolaridad.

En la ciudad de Chachapoyas, hay un elevado número de pacientes que consultan por faringoamigdalitis y se desconoce el agente causal, pero a todos se les trata con penicilina. Hay presencia clínica de pacientes que manifiestan signos y síntomas de infecciones secundarias no supurativas, que podrían deberse a Streptococcus pyogenes.

\section{MÉTODOS}

En la consulta externa del Hospital I Higos Urco, EsSalud, se seleccionó los pacientes con cuadro clínico compatible con faringoamigdalitis aguda (eritematosas y congestivas) y, si no estaban recibiendo antibiótico, fueron incluidos voluntariamente al estudio.

Se tomó las muestras con hisopos y se las colocó en tubo plástico que contenía el medio de transporte Amies con carbón (Difco). Se las mantuvo a medio ambiente hasta su envío al Instituto de Medicina Tropical Daniel A. Carrión, lo cual se hacía preferentemente cada fin de semana. El tiempo que transcurrió desde que se tomó las muestras hasta que fueron procesadas osciló entre 3 y 18 días, pero la mayoría de las muestras fueron cultivadas dentro de los 7 días.

Las muestras fueron sembradas en agar Columbia-sangre de carnero, agar Columbia sangre de carnero con gentamicina, agar selectivo para Moraxella catarrhalis, agar manitol salado, Hitchens Pike modificado para concentrar estreptococos, y después de 24 horas a $37^{\circ} \mathrm{C}$ fueron resembradas con el método de la placa vertida, en agar Columbia sangre de carnero. También se empleó agar Sabouraud glucosado

A los Streptococcus beta hemolíticos aislados se les hizo antibiograma, por el método de disco-difusión estandarizado, sensibilidad a la bacitracina y serotipo.

\section{RESULTADOS}

Se procesó 148 muestras de secreción faringoamigdaliana, de pacientes de diferentes edades (Tabla 1), de los cuales 73 
Tabla 1. Grupos de edad.

\begin{tabular}{lr}
\hline Grupo de edad (años) & \multicolumn{1}{c}{$\mathrm{N}^{0}$} \\
\hline Menos de 5 & $41(27,7 \%)$ \\
5 a 10 & $50(33,8 \%)$ \\
11 a 17 & $32(21,6 \%)$ \\
Más de 18 & $25(16,9 \%)$ \\
Total & $148(100 \%)$ \\
\hline
\end{tabular}

correspondían al sexo masculino $(50,7 \%)$ y 75 (49,3\%), al femenino.

Un primer grupo de 89 muestras fueron procesadas del 11 de agosto al 15 de setiembre de 2004 y un segundo grupo de 59 muestras, del 5 al 29 de enero de 2005.

Resultaron negativas a bacterias patógenas cultivables $32(21,6 \%)$.

Las bacterias aisladas fueron:

1. Enterobacterias $57(49,1 \%)$

2. Bacilos Gram negativos no fermentadores

3. Staphylococcus aureus $21(18,1 \%)$

4. Moraxella catarrhalis $6(5,2 \%)$

5. Streptococcus pneumoniae $3(2,6 \%)$

6. Streptococcus beta hemolítico del grupo B

7. Streptococcus beta hemolítico del grupo A

8. Streptococcus beta hemolítico del grupo $G$ $1(0,9 \%)$

9. Candida albicans $20(17,2 \%)$

10. Candida spp. (no albicans) 8 ( $6,9 \%)$

En algunos casos se aisló más de una bacteria patógena.

Los 5 casos de los cuales se aisló estreptococos beta hemolíticos son detallados a continuación:

1. Caso $\mathrm{N}^{\circ} 7$ : se le tomó la muestra el 11-08-04 y se procesó a los 5 días. Era del sexo femenino, de 15 años de edad. Procedía de Huambo-Rodríguez de Mendoza y se le aisló Streptococcus pneumoniae, enterobacterias y Streptococcus beta hemolítico del grupo B (S. agalactiae), que resultó resistente a la bacitracina y en el antibiograma, resistente a cotrimoxazol y rifampicina, intermedio a ciprofloxacina y sensible a penicilina, eritromicina, vancomicina, cloranfenicol, lincomicina, tetraciclina, cefalotina, cefuroxima, cefotaxima y ceftriaxona.

2. Caso $\mathrm{N}^{\circ} 10$ : se le tomó la muestra el 09-08-04 y se procesó a los 7 días. Era del sexo masculino, de 9 años de edad. Procedía de San Francisco del Yeso (Luya) y se le aisló Streptococcus beta hemolítico del grupo A (S. pyogenes), que resultó sensible a bacitracina (20 $\mathrm{mm}$ ) y en el antibiograma fue resistente a cotrimoxazol y sensible a penicilina, vancomicina, ceftriaxona, eritromicina, tetraciclina, cefalexina, cefuroxima, cefotaxima, ciprofloxacina, cloranfenicol, lincomicina y rifampicina.

3. Caso $\mathrm{N}^{\circ} 25$ : se le tomó la muestra el 1108-04 y se procesó a los 5 días. Era del sexo femenino, de 5 años de edad. Procedía de Montevideo (Chachapoyas) y se le aisló Streptococcus beta hemolítico del grupo B (S. agalactiae), que resultó resistente a la bacitracina y en el antibiograma fue resistente a cotrimoxazol y lincomicina, intermedio a eritromicina, tetraciclina y ciprofloxacina; y sensible a penicilina, vancomicina, cloranfenicol, ceftriaxona, cefuroxima, cefalexina, cefotaxima y rifampicina.

4. Caso $\mathrm{N}^{\circ}$ 28: se le tomó la muestra el 09-08-04 y se procesó a los 7 días. Era del sexo femenino, de 25 años de edad. Procedía de Oyeros (Chachapoyas) y se le aisló Streptococcus beta hemolítico del grupo B (S. agalactiae), que resultó resistente a la bacitracina y en el antibiograma fue resistente a cotrimoxazol, intermedio a tetraciclina y sensible a penicilina, lincomicina, eritromicina, ciprofloxacina, vancomicina, cloranfenicol, rifampicina, ceftriaxona, cefuroxima, cefalexina y cefotaxima.

5. Caso $\mathrm{N}^{\circ}$ 101: se le tomó la muestra el 17-01-05 y se procesó a los 7 días. Era del sexo femenino, de 5 años de edad. Procedía de Chachapoyas y se le aisló enterobacterias y Streptococcus beta hemolítico del grupo $\mathrm{G}$, que resultó resistente a la bacitracina y en el antibiograma fue sensible a todos los antibióticos testados.

En las Tablas 2 y 3 podemos apreciar esquemáticamente los 5 casos positivos a estreptococos beta hemolíticos.

\section{DISCUSIÓN}

La faringoamigdalitis aguda es una infección causada principalmente por el Streptococcus beta hemolítico del grupo A (Streptococcus pyogenes), para lo cual se administra empíricamente penicilina.

En Chachapoyas, hay una numerosa concurrencia de pacientes con este padecimiento, especialmente niños, y sorpresivamente los mayores aislamientos de bacterias cultivables, porque no buscamos Chlamydia pneumoiae ni Mycoplasma pneumoniae, fueron las enterobacterias $(49,1 \%)$.

Solo 5 Streptococcus beta hemolíticos fueron aislados en nuestro estudio, a pesar de haber comprobado previamente la supervivencia del Streptococcus pyogenes en el medio de transporte utilizado, de por lo menos 7 días, tiempo en el cual se procesó la mayoría de las muestras.

Se tuvo un solo caso de Streptococcus pyogenes, aislado de un niño de 9 años, a pesar de que en otros países, como México, Casani y col. ${ }^{(1)}$ observaron que se han incrementado las infecciones por este microorganismo, en las últimas 2 décadas, mientras que en nuestro país no hemos encontrado publicaciones al respecto. En Argentina ${ }^{(2)}$ y Venezuela ${ }^{(3,4)}$, bajo aislamiento. La cepa resultó sensible a los betalactámicos, macrólidos y lincosamidas, pero se comunica que siempre son sensibles a la penicilina y otros betalactámicos, mientras han aparecido resistencia a los macrólidos y lincosamidas $(5,6)$.

Streptococcus agalactiae o Streptococcus beta hemolítico del grupo B es la principal causa que amenaza la vida a los recién nacidos y es responsable de abortos en mujeres y neumonía, sepsis y meningitis en recién nacidos. Nosotros lo hemos encontrado en 3 pacientes; pero, Lo y col. ${ }^{(7)}$ refieren que puede colonizar la faringe de lactantes y niños y es muy raro asociarlo con infección en espacio retrofaríngeo; en el caso descrito por ellos sugieren que el Streptococcus del grupo B debería también ser considerado como un potencial causante de absceso retrofaríngeo en el grupo pediátrico, más allá de los neonatos. Fue el serotipo más aislado en el estudio hecho en Venezuela (3).

Los Streptococcus beta hemolíticos del grupo $G$ fueron descritos por primera vez por Lancefield y Hare, en 1935, y considerados como no patógenos en humanos. Sin embargo, Sylvetsky y col. ${ }^{(8)}$ refieren haberse descrito infecciones serias causadas

Tabla 2. Datos de filiación y diagnóstico.

\begin{tabular}{|c|c|c|c|c|}
\hline $\mathrm{N}^{0}$ caso & Procedencia & Sexo & Edad & Diagnóstico \\
\hline 7 & $\begin{array}{l}\text { Huambo-Rodríguez } \\
\text { de Mendoza }\end{array}$ & $\mathrm{F}$ & 15 & $\begin{array}{l}\text { Streptococcus pneumoniae } \\
\text { Streptococcus del grupo B } \\
\text { Enterobacteria }\end{array}$ \\
\hline 10 & $\begin{array}{l}\text { San Francisco } \\
\text { del Yeso (Luya) }\end{array}$ & M & 9 & Streptococcus del grupo A \\
\hline 25 & $\begin{array}{l}\text { Montevideo } \\
\text { (Chachapoyas) }\end{array}$ & $\mathrm{F}$ & 5 & Streptococcus del grupo B \\
\hline 28 & $\begin{array}{l}\text { Oyeros } \\
\text { (Chachapoyas) }\end{array}$ & $\mathrm{F}$ & 25 & Streptococcus del grupo B \\
\hline 101 & Chachapoyas & $\mathrm{F}$ & 5 & $\begin{array}{l}\text { Streptococcus del grupo G } \\
\text { Enterobacteria }\end{array}$ \\
\hline
\end{tabular}


Tabla 3. Susceptibilidad del Streptococcus beta hemoliticos.

\begin{tabular}{lccccc}
\hline Antibiótico & $\begin{array}{c}\text { Caso 7 } \\
\text { S. grupo B }\end{array}$ & $\begin{array}{c}\text { Caso 10 } \\
\text { S. grupo A }\end{array}$ & $\begin{array}{c}\text { Caso 25 } \\
\text { S. grupo B }\end{array}$ & $\begin{array}{c}\text { Caso 28 } \\
\text { S. grupo B }\end{array}$ & $\begin{array}{c}\text { Caso } 101 \\
\text { S. grupo G }\end{array}$ \\
\hline Cefalexina & S & S & S & S & S \\
Cefotaxima & S & S & S & S & S \\
Ceftriaxona & S & S & S & S & S \\
Cloranfenicol & S & S & S & S & S \\
Cefuroxima & S & S & S & S & S \\
Ciprofloxacina & I & S & I & S & S \\
Cotrimoxazol & R & R & R & R & S \\
Eritromicina & S & S & I & S & S \\
Lincomicina & S & S & R & S & S \\
Penicilina & S & S & S & S & S \\
Rifampicina & R & S & S & S & S \\
Tetraciclina & S & S & I & I & S \\
Vancomicina & S & S & S & S & S \\
\hline
\end{tabular}

$S=$ sensible, $\mathrm{l}=$ intermedio, $\mathrm{R}=$ resistente.

por el Streptococcus del grupo G, incluyendo piel y tejidos blandos, otitis media, faringitis, neumonía, meningitis, peritonitis, endocarditis, artritis y sepsis puerperal y neonatal, y han observado un incremento en las bacteriemias, que sobrepasa a las causadas por Streptococcus pyogenes, sobre todo en personas mayores con infección de la piel. Isaacson y col. ${ }^{(9)}$ refieren que el Streptococcus del grupo $\mathrm{G}$ ha sido reconocido como un importante organismo invasivo en la última década y que la faringitis causada por este microorganismo ha sido hallada en hospederos normales, describiendo un caso de epiglotitis y añadiendo que estas bacterias son muy sensibles a la penicilina y cefalosporinas, usadas empíricamente en el tratamiento de la epiglotitis. Nosotros encontramos un caso con esta bacteria y fue sensible a la penicilina y cefalosporinas. Se ha comunicado su presencia en Venezuela ${ }^{(3,4)}$ y Argentina ${ }^{(2)}$.

En conclusión, las enterobacterias fueron las bacterias más aisladas en la faringoamigdalitis aguda en Chachapoyas; pero, no podemos descartar definitivamente al Streptococcus pyogenes, porque esta bacteria puede refugiarse en el interior de las amígdalas, lo que dificulta su aislamiento ${ }^{(10)}$. Sería recomendable complementar el estudio con el dosaje de antiestreptolisina $\mathrm{O}$, para estar seguro de su ausencia.

El empleo del disco de bacitracina en el diagnóstico diferencial de los Streptococcus beta hemolíticos es de gran ayuda para los laboratorios que no cuentan con el kit para hacer la serotipificación y así puedan diagnosticar por lo menos Streptococcus pyogenes, que fue también la única sensible en el estudio.

Las 5 cepas de Streptococcus beta hemolíticos que aislamos fueron sensibles a los betalactámicos, macrólidos y lincosamidas; pero, su pequeño número no nos permite generalizar los hallazgos.

\section{AGRADECIMIENTO}

A la Facultad de Medicina de la Universidad Nacional Mayor de San Marcos, por su apoyo económico en la realización del estudio, dentro del III Concurso de proyectos de investigación.

\section{REFERENCIAS BIBLIOGRÁFICAS}

1. Casaní C, Morales M, Santos M, Otero MC, Pérez D, Asensi F. Estudio epidemiológico de un brote de escarlatina. Revista de Pediatría de Atención Primaria. 2001;3(8):41-9.

2. Villar HE, Jugo MB, Santana G, Baserni M, Reil JM. Aumento de la prevalencia de Estreptococos beta hemolíticos en hisopados faríngeos en Buenos Aires. Medicina (Buenos Aires). 2005;65:311-4.

3. Romero S, Ginestre M, Rincón G, Harris B, Martínez A. Streptococcus betalactámicos en la orofarínge de escolares asintomáticos de dos instituciones del estado de Zula. Rev Soc Ven Microbiol. 2002;22(1):1-9.

4. Romero S, Ginestre M, Martínez A, Rincón G, Harris B, Castellano M. Estreptococos betahemolíticos en la faringe de personal militar. Rev Soc Ven Microbiol. 2001;21(2):1-4.

5. Gonzales A, Ortiz C, Mota R, Dickinson E, Dávila $R$, Fernández MA. Sensibilidad antimicrobiana y caracterización de cepas de Streptococcus pyogenes aisladas de un brote de escarlatina. Salud Pública de México. 2002;44(5):437-41.

6. Rodríguez RS, Calderón E, Gómez D, Espinoza LE. Características de la resistencia antimicrobiana de una colección clínica de Streptococcus pyogenes. Salud Pública de México. 2000;42(3):226-9.

7. Lo WT, Lien YM, Wang CC, Chu ML. Retropharyngeal abscess caused by Grupo B Streptococcus in a previously healthy child. Infection. 2001;29(5):289-90.

8. Sylvetsky N, Raveh D, Schlesinger Y, Rudensky B, Yinnon AM. Bacteremia due to beta-hemolytic Streptococcus Grupo G: Increasing incidence and clinical characteristics of patients. Am J Med. 2002;112:622-6.

9. Isaacson G, Isaacson DM. Pediatric epiglottitis caused by Group G beta-hemolytic Streptococcus. Ped Inf Dis J. 2003;22(9):846-7.

10. Kasenomm P, Piirson A, Kull M Jr, Mikelsaar M. Amigdalectomía en adultos con amigdalitis recurrente. BMC Ear Nose Throat Disord. 2005;5:7.

Manuscrito recibido el 10 de marzo de 2008 y aceptado para publicación el 13 de mayo de 2008.

Correspondencia:

Dr. José María Guevara Duncan

Instituto de Medicina Tropical Daniel A. Carrión

Jr. José Santos Chocano 199

Callao 02, Perú

Correo-e: jguevarad@unmsm.edu.pe 